\title{
SOME PROBLEMS IN PARTITIO NUMERORUM
}

\author{
P. ERDÖS and J. H. LOXTON
}

(Received 24 April 1977)

Communicated by J. Pitman

\begin{abstract}
We consider some unconventional partition problems in which the parts of the partition are restricted by divisibility conditions, for example, partitions $n=a_{1}+\ldots+a_{k}$ into positive integers $a_{1}, \ldots, a_{k}$ such that $a_{1}\left|a_{2}\right| \ldots \mid a_{k}$. Some rather weak estimates for the various partition functions are obtained.
\end{abstract}

Subject classification (Amer. Math. Soc. (MOS) 1970): 10 A 45, $10 \mathrm{~J} 20$.

\section{Introduction}

In this paper, we shall consider various partition problems in which the parts of the partitions are restricted by divisibility conditions. Most of our remarks concern the following two situations:

(i) 'Chain partitions', that is partitions $n=a_{1}+\ldots+a_{k}$ into positive integers $a_{1}, \ldots, a_{k}$ such that $a_{1}\left|a_{2}\right| \ldots \mid a_{k}$.

(ii) 'Umbrella partitions', that is partitions into positive integers such that every part divides the largest one. Our aim is to estimate the partition functions which arise in each case for partitions with distinct parts and for partitions in which repetitions are allowed.

This work arose from a question of R. W. Robinson about chain partitions with repetitions which, in turn, came from attempts to count a certain kind of tree. This particular partition problem is closely connected with $m$-ary partitions, that is partitions as sums of powers of a fixed integer $m$, which are obvious instances of the types of partitions described above. In another direction, the problem of representing numbers by umbrella partitions has some connections with the 'practical numbers' of Srinivasan.

We would like to thank Dr. B. Richmond for criticisms which have helped us to remove some of the obscurities in our original manuscript. 
Throughout the paper, $c$ denotes a generic positive constant, not necessarily the same at each occurrence, and $\log$ and $\log _{2}$ denote logarithms to base $e$ and base 2 respectively.

\section{Chain partitions with distinct parts}

Let $p(n)$ be the number of partitions $n=a_{1}+\ldots+a_{k}$ into distinct positive integers $a_{j}$ with $a_{1}\left|a_{2}\right| \ldots \mid a_{k}$ and let $p_{1}(n)$ be the number of partitions of this type with $a_{1}=1$. Clearly,

$$
p(n)=p_{1}(n)+p_{1}(n+1)
$$

and, by considering the partitions of $n$ with $a_{1}=1$ and $a_{2}=d$ and summing over the possible values of $d$, we find the recurrence

$$
p_{1}(n)=\sum_{d \mid n-1, d>1} p_{1}((n-1) / d)=\sum_{d \mid n-1, d<n-1} p_{1}(d) .
$$

It is easy to see that $p(n)$ tends to infinity with $n$, and even a little more.

THEOREM 1. For $n \geqslant 6$, we have $p(n) \geqslant \log _{2} n$. For $n \geqslant 27$, we have $p_{1}(n) \geqslant \frac{1}{2} \log _{2} n$, except when $n-1$ is prime, in which case $p_{1}(n)=1$.

PROOF. First consider $p(n)$. For each integer $k$ with $1 \leqslant 2^{k} \leqslant n / 3$, we can choose an odd integer $t$ satisfying $n-2^{k+1}<2^{k} t \leqslant n$ and we obtain one of the partitions counted in $p(n)$ by writing $n-2^{k} t$ in the binary scale, that is using some or all of the parts $1,2,2^{2}, \ldots, 2^{k}$, and adding the last part $2^{k} t$. If we also count the partition arising from the binary representation of $n$, we have

$$
p(n) \geqslant \log _{2}[n / 3]+2 \geqslant \log _{2} n,
$$

for $n \geqslant 6$. The same argument shows that the inequalities (3) also hold for $p_{1}(n)$ for odd $n$. Finally, if $n$ is even and $n-1$ is composite, then $n-1$ has an odd proper divisor $d \geqslant(n-1)^{\ddagger}$ and by (2) and what we have already proved

$$
p_{1}(n) \geqslant p_{1}(d)+1 \geqslant \frac{1}{2} \log _{2}(n-1)+1 \geqslant \frac{1}{2} \log _{2} n,
$$

for $n \geqslant 27$, as required.

We can say rather more about the sum functions of the partition functions $p(n)$ and $p_{1}(n)$. Since they behave similarly, by (1), we introduce only

$$
P_{1}(x)=\sum_{1 \leqslant n \leqslant x} p_{1}(n)
$$


By considering the partitions of $n$ with $a_{1}=1$ and $a_{2}=d$ and summing over $d$, we get

$$
P_{1}(x)=\sum_{2 \leqslant d \leqslant x-1} P_{1}((x-1) / d)+1 .
$$

This functional equation for $P_{1}(x)$ is essentially the same as a functional equation arising in the theory of 'factorisatio numerorum'. (See Kalmár (1931), Hille (1936), Erdös (1941) and Grosswald (1974).) Denote by $f(n)$ the number of representations of $\boldsymbol{n}$ as a product of factors greater than 1 . Here, two representations are considered identical if and only if they have the same factors in the same order. We define $f(1)=1$. By arguments like those used above, we get

$$
f(n)=\sum_{d \mid n, d<n} f(d)
$$

and, for the sum function of the $f(n)$,

$$
F(x)=\sum_{1 \leqslant n \leqslant x} f(n)=\sum_{2 \leqslant d \leqslant x} F(x / d)+1 .
$$

Starting from (6), Erdös (1941) gave an elementary proof that $F(x) \sim c x^{\rho}$ as $x \rightarrow \infty$, where $\rho$ is the unique positive root of $\zeta(\rho)=2$. With a few minor modifications, this method yields the following result for $P_{1}(x)$.

THEOREM 2. Let $\rho$ be the unique positive root of $\zeta(\rho)=2$, where $\zeta(s)$ is the Riemann zeta function. Then $P_{1}(x) \sim c x^{\rho}$ as $x \rightarrow \infty$.

For the factorization problem, the standard machinery of analytic number theory is also available. Indeed, from (5), $f(n)$ has the generating Dirichlet series

$$
\sum_{n=1}^{\infty} \frac{f(n)}{n^{s}}=\frac{1}{2-\zeta(s)}
$$

and so, by the Wiener-Ikehara Tauberian theorem,

$$
F(x)=-\frac{x}{\rho \zeta^{\prime}(\rho)}+\Omega_{\diamond}\left(x^{\rho-s}\right)
$$

as $x \rightarrow \infty$, for every $\varepsilon>0$. (See Hille (1936).) For our partition problem, we have not been able to evaluate the constant $c$ in Theorem 2, but we can still show that

$$
P_{1}(x)=c x^{\rho}+\Omega_{\varepsilon}\left(x^{\rho-s}\right)
$$

as $x \rightarrow \infty$, for every $\varepsilon>0$. The assertion (7) is clearly true if $\lim \sup p_{1}(n) / n^{\rho-e}>0$. On the other hand, if $p_{1}(n) \leqslant c(\varepsilon) n^{\rho-\varepsilon}$, for some positive constant $c(\varepsilon)$, and we 
introduce the Dirichlet series

$$
\varphi(s)=\sum_{n=1}^{\infty} \frac{p_{1}(n)}{n^{8}}, \quad \psi(s)=\sum_{n=1}^{\infty} \frac{p_{1}(n+1)}{n^{8}},
$$

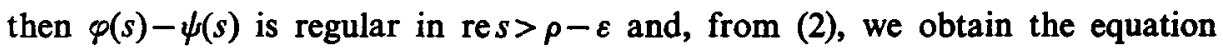
$\varphi(s)+\psi(s)=\zeta(s) \varphi(s)$, that is

$$
\varphi(s)=\{\varphi(s)-\psi(s)\} /\{2-\zeta(s)\}
$$

Thus $\varphi(s)$ has a meromorphic continuation to the half plane re $s>\rho-\varepsilon$ and it is an almost periodic function of im $s$ in this region since both the factors on the right in the above equation have this property. Next, $\{2-\zeta(s)\}^{-1}$ has a simple pole at $s=\rho$ and, by Theorem $2, \varphi(s)$ has some sort of singularity at $s=\rho$, so $\varphi(s)$ must have a simple pole at $s=\rho$ and, by almost periodicity, it has poles in every vertical strip $\rho-\delta<\operatorname{res}<\rho(0<\delta<\varepsilon)$. Consequently, the assertion (7) holds in this case as well.

We are unable to obtain accurate upper and lower estimates for the functions $p(n)$ and $p_{1}(n)$ beyond the results of Theorems 1 and 2 . Computations of these functions for $n \leqslant 10000$ suggest that the upper bound $p(n)<c(\varepsilon) n^{\rho-1+s}$ might be true for every $\varepsilon>0$. If so, this would be in marked contrast to the behaviour of the factorization function $f(n)$ for which it is known that $\lim \sup f(n) / n^{\rho-s}>0$ for every $\varepsilon>0$. (See Hille (1936) and Erdös (1941).) In view of the naïveté of Theorem 1, it seems likely that $p(n) / \log n \rightarrow \infty$ as $n \rightarrow \infty$, but the rather limited experimental data mentioned above do not convincingly confirm or contradict this.

In this connection, it may be observed that if the numbers $n$ and $2 n+1$ are both prime, then $p(2 n+1)=p(n)+1$ and, indeed, if the numbers $n_{j}=2^{j}(n+1)-1$ $(0 \leqslant j \leqslant k)$ are all prime, then $p\left(n_{k}\right)=p(n)+k$. So the problem of estimating $p(n)$ from below is inextricably bound up with the problem of estimating the least composite number in a sequence of the shape $m_{j}=2^{j} m-1(j \geqslant 0)$. We cannot prove very much about these sequences unconditionally. However, if we assume the truth of Artin's conjecture that the number of primes less than $x$ for which 2 is a primitive root is asymptotically $c^{-1} x / \log x$ as $x \rightarrow \infty$, then we can show that the least composite $m_{j}$ is less than $m^{c+1+8}$ for large $m$ and any $\varepsilon>0$. For, we can choose a prime $p<(c+\varepsilon) \log m$ such that $p \nmid m$ and 2 is a primitive root $\bmod p$ and we observe that one term in every $p$ consecutive terms of the sequence $\left\{m_{j}\right\}$ is a multiple of $p$. Indeed, Hooley has shown under hypotheses similar to those which he used to prove Artin's conjecture that almost all the terms in a sequence of this type are composite. (See Hooley (1976), Chapters 3 and 7.) These conditional results do not seem to give any improvement on Theorem 1 . We can show unconditionally only that the least composite number $m_{j}=2^{j} m-1$ is less than $2^{m / 4} m$, providing $m>6$. For, if $p$ is an odd prime divisor of $m-2^{l}$ for some $l$, then 
$m_{k(p-1)-l}$ is composite whenever $k>l /(p-1)$ because

$$
m_{k(p-1)-l}=2^{k(p-1)-l} m-1 \equiv 2^{k(p-1)}-1 \equiv 0 \quad(\bmod p) .
$$

Now, if $m>12$ and $m_{0}=m-1$ is prime, it can be seen that one of the numbers $m-2, m-4, m-8$ has an odd prime divisor less than $m / 4$ and so we have a composite $m_{j}$ less than $2^{m / 4} m$, as asserted.

We remark on another connection between chain partitions and the factorization problem. Let $p(m, n)$ be the number of partitions $n=a_{1}+\ldots+a_{k}$ into distinct positive integers $a_{j}$ with $a_{1}\left|a_{2}\right| \ldots\left|a_{k}\right| m$ and let $p_{1}(m, n)$ be the number of partitions of this type with $a_{1}=1$. As before,

$$
p(m, n)=p_{1}(m, n)+p_{1}(m, n+1) .
$$

To each factorization $d=a_{1} a_{2} \ldots a_{k}$ into factors greater than 1 , we make correspond the partition $n=1+a_{1}+a_{1} a_{2}+\ldots+a_{1} a_{2} \ldots a_{k}$. On counting the number of partitions and factorizations which arise as $d$ runs through all the divisors of $m$ less than $m$, we get the equation

$$
\sum_{n=1}^{m-1} p_{1}(m, n)=\sum_{d \mid m, d<m} f(d)=f(m) .
$$

This suggests the related question of estimating how many numbers $n$ with $1 \leqslant n \leqslant m$ can be represented by a partition $n=a_{1}+\ldots+a_{k}$ into distinct positive integers $a_{j}$ with $a_{1}\left|a_{2}\right| \ldots\left|a_{k}\right| m$. Clearly, the number of representable numbers is less than $m$ unless $m$ is a power of 2 . Moreover, if $m=p_{1} p_{2} \ldots p_{h}$ is the product of $h$ primes, not necessarily distinct, then there are at least $2^{h}$ representable numbers, namely the numbers $\varepsilon_{0}+\varepsilon_{1} p_{1}+\varepsilon_{2} p_{1} p_{2}+\ldots+\varepsilon_{h-1} p_{1} p_{2} \ldots p_{h-1}\left(\varepsilon_{j}=0\right.$ or 1$)$. However, if $m$ is the product of the first $h$ primes, say, we cannot decide whether there are as many $m^{\delta}$ representable numbers for some $\delta>0$.

\section{Chain partitions with repetitions}

Let $q(n)$ be the number of partitions $n=a_{1}+\ldots+a_{k}$ into positive integers $a_{j}$ with $a_{1}\left|a_{2}\right| \ldots \mid a_{k}$, repetitions being allowed. Arguing as before, we find

$$
q(n)=\sum_{d \mid n} q((n-d) / d)=\sum_{d \mid n} q(d-1),
$$

with the convention that $q(0)=1$. Included among the partitions counted in $q(n)$ are the binary partitions of $n$, that is the partitions of $n$ as sums of powers of 2; we denote the number of these by $b(n)$. The function $b(n)$ satisfies the recurrence

$$
b(2 n+1)=b(2 n), \quad b(2 n)=b(2 n-1)+b(n) \quad(n \geqslant 1),
$$


and we have

$$
B(x)=\sum_{0 \leqslant n \leqslant x} b(n)=b(2[x])
$$

For $B(x)$, de Bruijn (1948) has proved the extremely precise asymptotic result

(10) $\log B(x)=\frac{1}{2 \log 2}\left(\log \frac{x}{\log x}\right)^{2}+\left(\frac{1}{2}+\frac{1}{\log 2}+\frac{\log \log 2}{\log 2}\right) \log x$

$$
-\left(1+\frac{\log \log 2}{\log 2}\right) \log \log x+U\left(\frac{\log x-\log \log x}{\log 2}\right)+o(1)
$$

as $x \rightarrow \infty$, where $U(t)$ is a certain periodic function with period 1 . In fact, he gives the periodic function $U(t)$ explicitly by its Fourier series and further analyses the structure of the $o(1)$ term. Our aim in this section is the following estimate for the sum function

$$
Q(x)=\sum_{0 \leqslant n \leqslant x} q(n)
$$

which parallels the above result for $B(x)$, except that we cannot give the oscillatory term explicitly.

THEOREM 3. We have

$$
\begin{aligned}
& \log Q(x)=\frac{1}{2 \log 2}\left(\log \frac{x}{\log x}\right)^{2}+\left(\frac{1}{2}+\frac{1}{\log 2}+\frac{\log \log 2}{\log 2}\right) \log x \\
&-\left(1+\frac{\log \log 2}{\log 2}\right) \log \log x+V\left(\frac{\log x-\log \log x}{\log 2}\right)+o(1)
\end{aligned}
$$

as $x \rightarrow \infty$, where $V(t)$ is a certain periodic function with period 1.

The proof of Theorem 3 is rather long and we proceed by means of a number of lemmas. We begin with a first approximation to Theorem 3 which shows that at least $b(n)$ accounts for a positive proportion of the partitions of $q(n)$.

LEMMA 1. We have $b(n) \leqslant q(n) \leqslant c b(n)$.

Proof. To prove the second inequality, we use (8) and induction. From (9), $b(n)$ is increasing, so by (9) again, we have

$$
\sum_{d \mid n} b(d-1) \leqslant b(n-1)+\sum_{d ! n, d \geqslant 2} b\left(\frac{n}{d}\right)=b(n)+\sum_{d \mid n, d \geqslant 3} b\left(\frac{n}{d}\right) .
$$


Write $b(n / d)=b(n) w(n, d)$. From (10), after a little calculation, we obtain

(12) $\log w(n, d) \leqslant-\frac{\log d \cdot \log n}{\log 2}+\frac{(\log d)^{2}}{2 \log 2}+\frac{\log d \cdot \log \log n}{\log 2}$

$$
+\left(\frac{1}{2}-\frac{\log \log 2}{\log 2}\right) \log d+O(1)
$$

as $n \rightarrow \infty$. Let $\delta$ be a positive number so small that the number $\eta$ defined by

$$
\eta=\frac{3}{4}-(1+\delta) \frac{\log 2}{\log 3}
$$

is also positive. For the rest of the argument, we assume that $n$ is a positive integer chosen sufficiently large so that all the inequalities are valid. If $d \leqslant n^{\frac{1}{2}}$, then from (12),

$$
\log w(n, d)<-\frac{\log d \cdot \log n}{\log 2}\left(\frac{3}{4}-\frac{\eta}{2}\right),
$$

by our choice of $n$, and so

$$
\sum_{d \mid n, 3 \leqslant d \leqslant n^{\frac{1}{t}}} w(n, d)<\exp \left\{-\frac{\log 3 \cdot \log n}{\log 2}\left(\frac{3}{4}-\eta\right)\right\}=n^{-1-\delta} .
$$

Again, if $d>n^{\frac{1}{2}}$, then

$$
\log w(n, d)<-\frac{(\log d)^{2}}{2 \log 2}<-\frac{(\log n)^{2}}{8 \log 2}
$$

and so

$$
\sum_{d \mid n, d>n^{\frac{1}{2}}} w(n, d)<\exp \left\{-\frac{(\log n)^{2}}{8 \log 2}+\log n\right\}<n^{-1-\delta} .
$$

Hence, from (11),

$$
\sum_{d \mid n} b(d-1)<b(n)\left\{1+O\left(n^{-1-\delta}\right)\right\}
$$

and, finally, by (8) and a simple induction, we have

$$
q(n) \leqslant b(n) \prod_{m \leqslant n}\left\{1+O\left(m^{-1-\delta}\right)\right\}<c b(n),
$$

as required.

Now let $N$ be a positive integer and define a sequence $\left\{a_{N}(n)\right\}$ by

and

$$
a_{N}(n)=q(n) \quad(0 \leqslant n<2 N)
$$

$$
a_{N}(2 n+1)=a_{N}(2 n), \quad a_{N}(2 n)=a_{N}(2 n-1)+a_{N}(n) \quad(n \geqslant N) .
$$


We show next in Lemma 2 that, for large $N, a_{N}(n)$ is a good approximation to $q(n)$. Then, to complete the proof of Theorem 3, we use a Tauberian theorem of Ingham (1941) to show that the sum function of $a_{N}(n)$ has the behaviour specified for $Q(x)$ in the theorem. A similar application of Ingham's Tauberian theorem was made by Pennington (1953) in discussing the binary partition function.

LEMMA 2. Given $\varepsilon>0$, there is a positive number $N_{1}(\varepsilon)$ such that

$$
a_{N}(n) \leqslant q(n)<(1+\varepsilon) a_{N}(n)
$$

for all $n$, whenever $N \geqslant N_{1}(\varepsilon)$.

Proof. By (8) and (9) and Lemma 1, we have

$$
b(n) \leqslant a_{N}(n) \leqslant q(n) \leqslant c b(n) .
$$

So, by the argument used in the proof of Lemma 1 , if $\delta>0$ is sufficiently small, we have

$$
q(n) \leqslant a_{N}(n) \prod_{2 N \leqslant m \leqslant n}\left\{1+O\left(m^{-1-\delta}\right)\right\},
$$

which gives the lemma.

The generating function of the $b(n)$ is

$$
f(z)=\sum_{n=0}^{\infty} b(n) z^{n}=\prod_{k=0}^{\infty}\left(1-z^{2^{k}}\right)^{-1} \quad(|z|<1) .
$$

We set $F(s)=f\left(e^{-s}\right)$ for re $s>0$. The following formula for $F(s)$ is due to de Bruijn (1948).

LEMMA 3. Let $0<\theta<\pi / 2$ and let $\Delta(\theta)$ be the sector $|\arg s| \leqslant \theta$. Then

$$
\log F(s)=\frac{(\log s)^{2}}{2 \log 2}-\frac{1}{2} \log s+W\left(-\frac{\log s}{\log 2}\right)+O(|s|),
$$

as $s \rightarrow 0$, uniformly in $\Delta(\theta)$. Here, $W(t)$ is a certain periodic function of period 1 ana $W(-\log s / \log 2)$ is bounded in $\Delta(\theta)$.

Now, we introduce the functions

$$
g_{n}(z)=\sum_{k=0}^{\infty} z^{2^{n}} \prod_{j=0}^{k}\left(1-z^{2}\right)^{-1} \quad(|z|<1 ; n=1,2, \ldots)
$$

and we set $G_{n}(s)=g_{n}\left(e^{-s}\right)$ for re $s>0$. 
LEMMA 4. Let $0<\theta<\pi / 2$ and let $\Delta(\theta)$ be as in Lemma 3. For each fixed $n$, we have

$$
G_{n}(s) \sim F(s) \exp \left\{W_{n}(-\log s / \log 2)\right\},
$$

as $s \rightarrow 0$, uniformly in $\Delta(\theta)$. Here, $W_{n}(t)$ is a periodic function of period 1 and $W_{n}(-\log s / \log 2)$ is bounded in $\Delta(\theta)$.

Proof. Let $\rho$ be the unique root of $\rho^{n}=1-\rho^{2}$ with $0<\rho<1$; $\rho$ has the asymptotic expansion

$$
\rho=1-\frac{\log n}{n}+\frac{\log \log n}{n}+O\left(\frac{1}{n}\right) .
$$

Suppose $0<x<1$ and choose $m$ to be the integer satisfying $x^{2 m} \leqslant \rho<x^{2^{m-1}}$. Finally, define $y$ by $\rho^{v}=x^{2 m}$. Thus, $1 \leqslant y<2$ and $\log y / \log 2$ is the fractional part of $\left(\log \log x^{-1}-\log \log \rho^{-1}\right) / \log 2$. We can now write

$$
\begin{aligned}
g_{n}(x)=\frac{f(x)}{f\left(\rho^{\nu}\right)}\left\{\sum_{k=1}^{m} \rho^{2-k} n y\right. & \left.\prod_{j=1}^{k-1}\left(1-\rho^{2^{-j} y}\right)+\sum_{k=0}^{\infty} \rho^{2^{k} n y} \prod_{j=0}^{k}\left(1-\rho^{2 y}\right)^{-1}\right\} \\
= & \frac{f(x)}{f\left(\rho^{\nu}\right)}\left\{\ldots+\rho^{n y / 2}+\frac{\rho^{n y}}{1-\rho^{\nu}}+\frac{\rho^{2 n y}}{\left(1-\rho^{\nu}\right)\left(1-\rho^{2 y}\right)}+\ldots\right\},
\end{aligned}
$$

where the terms shown are, in fact, the biggest terms of the series. In the first tail the common ratios of consecutive terms are

$$
\rho^{2^{-k-1} n \nu}\left(1-\rho^{2^{-k} y}\right)<1-\rho^{y / 2}=O\left(\frac{\log n}{n}\right),
$$

by (13), and in the second tail, they are

$$
\frac{\rho^{2^{k} n y}}{1-\rho^{2^{2+1} n y}}<\frac{\rho^{2 n y}}{1-\rho^{4 \nu}}=\frac{\left(1-\rho^{2}\right)^{2 y}}{1-\rho^{4 y}}=O\left(\frac{\log n}{n}\right),
$$

so the series is dominated in both directions by a geometric series with common ratio $O(\log n / n)$. Further, by (13) and Lemma 3,

$$
\begin{aligned}
\log f\left(\rho^{\nu}\right)=\frac{1}{2 \log 2}\left(\log \frac{n}{\log n}\right)^{2} & +\left(\frac{1}{2}-\frac{\log y}{\log 2}\right) \log n \\
& +\left(\frac{1}{\log 2}-\frac{1}{2}+\frac{\log y}{\log 2}\right) \log \log n+O(1),
\end{aligned}
$$

Is $n \rightarrow \infty$, uniformly in $y$ in the interval $1 \leqslant y \leqslant 2$. This proves the assertions of the emma for real $s$ and they follow for complex $s$ since all the functions involved are egular in $\Delta(\theta)$. 
The generating function of the $a_{N}(n)$ is

$$
f_{N}(z)=\sum_{n=N}^{\infty} a_{N}(n) z^{n}=\sum_{k=0}^{\infty} h_{N}\left(z^{2^{k}}\right) \prod_{j=0}^{k}\left(1-z^{2}\right)^{-1} \quad(|z|<1),
$$

where

$$
h_{N}(z)=\sum_{n=N}^{2 N-1}\{q(n)-q(n-1)\} z^{n}+q(N-1) z^{N}
$$

This can be most easily seen by observing that $f_{N}(z)$ is the unique solution of the functional equation $f_{N}\left(z^{2}\right)=(1-z) f_{N}(z)-h_{N}(z)$ which is regular at the origin and vanishes there. We write $F_{N}(s)=f_{N}\left(e^{-8}\right)$ for re $s>0$. By Lemma 4,

$$
F_{N}(s) \sim F(s) \exp \left\{X_{N}(-\log s / \log 2)\right\}
$$

as $s \rightarrow 0$, uniformly in the sector $\Delta(\theta)$, for each $\theta$ with $0<\theta<\pi / 2$ and for each fixed $N$. Again, $X_{N}(t)$ is a periodic function with period 1 and $X_{N}(-\log s / \log 2)$ is bounded in $\Delta(\theta)$. Set

$$
A_{N}(x)=\sum_{N \leqslant n \leqslant x} a_{N}(n)
$$

We follow Pennington (1953) in applying a Tauberian theorem of Ingham to the transform

$$
F_{N}(s)=\int_{0}^{\infty} e^{-8 x} d A_{N}(x)
$$

Indeed, by (14) and Lemma 3, the functions $F(s)$ and $F_{N}(s)$ have the same asymptoti behaviour as $s \rightarrow 0$, so the details of the Tauberian argument for $F_{N}(s)$ are exactly the same as those for $F(s)$ given in Pennington (1953), pp. 540-544. Consequently $\log A_{N}(x)$ has the same asymptotic behaviour as $\log B(x)$ for large $x$, that is $\log A_{N}(x$. has the shape specified in Theorem 3. The theorem follows from this remark anc Lemma 2. For, given $\varepsilon>0$, we can choose $N$ so large that $\log Q(x)$ is approximater to within $\varepsilon / 2$ by $\log A_{N}(x)$ for all large $x$. Hence $\log Q(x)$ satisfies the estimate o Theorem 3 with error at most $\varepsilon$, say, for all large $x$ and this gives the required result

Our methods could be used to give bounds for the oscillation of $Q(x) / B(x)$ fo large $x$, but we have not been able to decide whether or not $Q(x) / B(x)$ approache a limit as $x \rightarrow \infty$. 


\section{Umbrella partitions with distinet parts}

Let $r(n)$ be the number of partitions of $n$ into distinct positive integers such that each part divides the largest one. As usual, we do not distinguish between partitions differing only in the order of their terms. We write

$$
D(n)=\max _{1 \leqslant m \leqslant n} d(m)
$$

where $d(m)$ denotes the number of positive divisors of the integer $m$. The next theorem gives an estimate for $r(n)$.

THEOREM 4. We have $\log \log r(n) \sim \log 2 . \log n / \log \log n$ as $n \rightarrow \infty$. More precisely, $\log r(n) \sim \log 2 . D(n)$ as $n \rightarrow \infty$.

ProOF. The second statement implies the first by the well-known estimate for the maximum order of $d(n)$. (See, for example, Hardy and Wright (1968), Theorem 317.)

We show first that $\log _{2} r(n) \leqslant\{1+o(1)\} D(n)$. Indeed, each partition of $r(n)$ with largest part $m$ corresponds to some subset of the divisors of $m$ which make up the terms of the partition. Since there are at most $n$ choices for the largest part $m$, this gives $r(n) \leqslant 2^{D(n)} n$, as asserted.

It remains to show that $\log _{2} r(n) \geqslant\{1+o(1)\} D(n)$, and for this we need some of the properties of the highly composite numbers of Ramanujan (1915). A positive integer $n$ is called highly composite if $d(m)<d(n)$ for every positive integer $m$ less than $n$. Now, given a positive integer $n$, let $m$ be the largest highly composite number not exceeding $n / 2$. By Ramanujan (1915), Section 28, we have $m \sim n / 2$ and $d(m) \sim D(n)$ as $n \rightarrow \infty$. Moreover, from Sections 8 and 23 of Ramanujan's paper, $m=2^{\alpha} 3^{\beta} 5^{\gamma} . .$. with $\alpha \geqslant \beta \geqslant \gamma \geqslant \ldots$ and $\alpha \sim \log \log n / \log 2(\log \log \log n)^{1}$. We choose a sequence $d_{1}=1<d_{2}<\ldots<d_{k}=m$ of divisors of $m$ with $d_{j} \leqslant 2 d_{j-1}(2 \leqslant j \leqslant k)$ and with $k$ as small as possible. Clearly, this can be done with $k<c \log n$. Now, let $D_{1}<D_{2}<\ldots$ be the consecutive divisors of $m$ excluding the $d_{j}$ and choose $r$ so that

$$
D_{1}+D_{2}+\ldots+D_{r} \leqslant m<D_{1}+D_{2}+\ldots+D_{r+1} \text {. }
$$

We assert that $r \sim d(m)$, for the number $2^{-\kappa} m$ with $\kappa=\left[(\log \log m)^{\mathbf{1}}\right]$ has $\{1+o(1)\} d(m)$ divisors whose sum is less than $m$. With this construction, we can write $n=m+s+t$ where $s$ is a sum of the $D_{j}$ 's and $t$ is a sum of the $d_{j}$ 's, giving a partition of the required sort for $n$. There are in all $2^{r}$ choices for $s$, so we have

as required.

$$
r(n) \geqslant 2^{\{1+o(1)\} d(m)} \geqslant 2^{\{1+o(1)\} D(n)},
$$


By analogy with the concluding remarks of Section 2, we can ask what positive integers $m$ enable us to represent all numbers $n$ with $1 \leqslant n \leqslant m$ by an umbrella partition with largest part dividing $m$. These are the 'practical numbers' of Srinivasan. Sierpinski (1955) showed that $m$ is a practical number if and only if it has the prime factorization $m=2^{\alpha_{0}} p_{1}^{\alpha_{1}} p_{2}^{\alpha_{2}} \ldots p_{h}^{\alpha_{h}}$ where $p_{1}<p_{2}<\ldots<p_{h}$ are odd primes, $\alpha_{0}, \alpha_{1}, \ldots, \alpha_{h}$ are positive integers and $p_{j+1} \leqslant 1+\sigma\left(2^{\alpha_{0}} p_{1}^{\alpha_{1}} p_{2}^{\alpha_{2}} \ldots p_{j}^{\alpha_{4}}\right)$ for $0 \leqslant j \leqslant h-1$. (Here, $\sigma(n)$ denotes, as usual, the sum of the positive divisors of $n$.) Consequently, the practical numbers have zero density. We can prove the stronger assertion that the density of integers $m$ for which a given integer $n$ is the sum of distinct divisors of $m$ tends to 0 as $n \rightarrow \infty$. The proof is omitted.

\section{Umbrella partitions with repetitions}

Finally, we consider the partition function $s(n)$ which is the number of partitions of $n$ into positive integers in which each part divides the largest part and repetitions are allowed. We obtain an estimate similar to the one in Section 4.

ThEOREM 5. We have $\log \log s(n) \sim \log 2 . \log n / \log \log n$ as $n \rightarrow \infty$. More precisely, $\log s(n) \sim \frac{1}{2} D(n) \log n$ as $n \rightarrow \infty$, where $D(n)$ is defined by (15).

Proof. Let $s(m, n)$ be the number of partitions of $n$ with largest part $m$ and with all parts dividing $m$. If $d$ divides $m$, then $d$ occurs at most $n / d$ times in any partition of $s(m, n)$ and, moreover, any choice for the divisors $d$ with each occurring at most $n / d$ times gives a partition of $s(m, n d(m))$. Hence

$$
s(m, n) \leqslant \prod_{d \mid m}([n / d]+1) \leqslant s(m, n d(m)) .
$$

From this,

$$
\begin{aligned}
s(n)=\sum_{m=1}^{n} s(m, n) & \leqslant \sum_{m=1}^{n} \prod_{d \mid m}\left(\frac{n}{d}+1\right) \\
& \leqslant \sum_{m=1}^{n}\left(\frac{n}{m^{1}}\right)^{d(m)} e^{\sigma(m) / n}<n^{\{t+o(1)\} D(n)},
\end{aligned}
$$

because, for any $\varepsilon>0$, the terms of the sum with $m<n^{1-\varepsilon}$ are absorbed in the o(1) error term. On the other hand,

$$
s(m, n d(m)) \geqslant \prod_{d \mid m} \frac{n}{d}=\left(\frac{n}{m^{i}}\right)^{d(m)},
$$


and so

$$
s(n) \geqslant \sum_{m=1}^{n} s(m,[n / d(m)]) \geqslant \sum_{m=1}^{n}\left([n / d(m)] / m^{\mathbf{b}}\right)^{d(m)}>n^{\{+o(1)\} D(n)}
$$

because, from Section 28 of Ramanujan (1915), there is a term of the sum with $m=D(n)$ and $m=\{1+o(1)\} n$. This proves the theorem.

\section{References}

N. G. de Bruijn (1948), 'On Mahler's partition problem', Proc. Kon. Nederl. Akad. Wet. (A) 51, 659-669.

P. Erdös (1941), 'On some asymptotic formulas in the theory of "factorisatio numerorum" ', Annals of Math. 42, 989-993. See also, Ann. of Math. 44 (1943), 648-651.

E. Grosswald (1974), 'Verallgemeinerte Eulersche Zahlen', Math. Z. 140, 173-177.

G. H. Hardy and E. M. Wright (1968). An introduction to the theory of numbers, 4th ed. (Oxford University Press).

E. Hille (1936), 'A problem in "factorisatio numerorum", ', Acta Arith. 2, 136-144.

C. Hooley (1976), Applications of sieve methods to the theory of numbers (Cambridge Tracts in Mathematics 70, Cambridge University Press).

A. E. Ingham (1941), 'A Tauberian theorem for partitions', Ann. of Math. 42, 1075-1090.

L. Kalmár (1931), 'Ủber die mittlere Anzahl der Produktdarstellungen der Zahlen. (Erste Mitteilung)', Acta Litt. ac Scient. Szeged 5, 95-107.

W. B. Pennington (1953), 'On Mahler's partition problem', Ann. of Math. 57, 531-546.

S. Ramanujan (1915), 'Highly composite numbers', Proc. London Math. Soc. (2) 14, 347-409.

W. Sierpinski (1955), 'Sur une propriété des nombres naturels', Annali di Mat. pura ed appl. (4) 39, 69-74.

Mathematical Institute

Hungarian Academy of Science

Budapest

Hungary
School of Mathematics

University of New South Wales

Kensington, N.S.W.

Australia 